\title{
Saccadic eye movements after low-dose oral alcohol exposure
}

\author{
Schmitt, K U ; Lanz, C ; Muser, M H ; Walz, F ; Schwarz, U
}

\begin{abstract}
OBJECTIVES: The influence of low-dose alcohol intake on reflexive visually guided saccades was investigated. METHODS: 40 healthy human subjects were orally given alcohol resulting in lowdose alcohol concentration (less than $0.8 \%$ ) blood alcohol concentration. Before and after exposure, horizontal saccadic eye movements were recorded at several points in time. The recordings were evaluated with regard to accuracy of the eye movements, latency, the peak eye velocity and the time constant that characterizes the main sequence. The results were compared to recordings from a reference group. RESULTS: It was found that the saccadic eye movement was clearly altered by low-dose alcohol intake. However, its characteristics were not unambiguously pathological when compared to the reference group, even though the subjects reported a moderate to strong effect of alcohol and there were clear signs of inebriation. CONCLUSIONS: The findings render the evaluation of saccades unsuitable as a simple test for the detection of low-dose alcohol intake.
\end{abstract}

DOI: https://doi.org/10.1016/j.jflm.2013.06.023

Posted at the Zurich Open Repository and Archive, University of Zurich

ZORA URL: https://doi.org/10.5167/uzh-85432

Journal Article

Accepted Version

Originally published at:

Schmitt, K U; Lanz, C; Muser, M H; Walz, F; Schwarz, U (2013). Saccadic eye movements after low-dose oral alcohol exposure. Journal of Forensic and Legal Medicine, 20(7):870-874.

DOI: https://doi.org/10.1016/j.jflm.2013.06.023 


\title{
Saccadic eye movements after low-dose oral alcohol
}

\section{exposure}

\author{
Kai-Uwe Schmitt ${ }^{1,2}$, Christian Lanz ${ }^{1}$, Markus H. Muser ${ }^{1}$, Felix Walz ${ }^{1}$, Urs Schwarz ${ }^{3}$
}

1: AGU - Working Group on Accident Mechanics, Zurich, Switzerland

2: Institute for Biomedical Engineering, University and ETH Zurich, Zurich, Switzerland

3: Department of Neurology, University Hospital Zurich, Zurich, Switzerland

Corresponding author:

Kai-Uwe Schmitt, PhD

AGU Zurich

Winkelriedstrasse 27

$\mathrm{CH} 8006$ Zurich

Switzerland

Telephone +41442515430

Fax +41442515431

E-mail schmitt@agu.ch

University and ETH Zurich

Institute for Biomedical Engineering

Gloriastrasse 35

$\mathrm{CH} 8092$ Zurich

Switzerland

Telephone $\quad+41446327536$

Fax +41446321193

E-mail_schmitt@ethz.ch

Co-authors:

Christian Lanz MD, Felix Walz MD, Markus Muser PhD

AGU Zurich

Winkelriedstrasse 27

$\mathrm{CH} 8006$ Zurich

Switzerland

Telephone $\quad+41442515430$

Fax +41442515431

E-mail_secretariat@agu.ch

Urs Schwarz MD

University Hospital Zurch

Dept. Neurology

Frauenklinikstrasse 26

$\mathrm{CH} 8006$ Zurich

Switzerland

E-mail us@usz.ch 


\section{ABSTRACT}

Objectives: The influence of low-dose alcohol intake on reflexive visually guided saccades was investigated.

Methods: 40 healthy human subjects were orally given alcohol resulting in low-dose alcohol concentration (less than $0.8 \%$ ) blood alcohol concentration. Before and after exposure, horizontal saccadic eye movements were recorded at several points in time. The recordings were evaluated with regard to accuracy of the eye movements, latency, the peak eye velocity and the time constant that characterizes the main sequence. The results were compared to recordings from a reference group.

Results: It was found that the saccadic eye movement was clearly altered by low-dose alcohol intake. However, its characteristics were not unambiguously pathological when compared to the reference group, even though the subjects reported a moderate to strong effect of alcohol and there were clear signs of inebriation.

Conclusions: The findings render the evaluation of saccades unsuitable as a simple test for the detection of low-dose alcohol intake.

Key words: low-dose alcohol intake, saccadic eye movement, fitness to drive, traffic medicine 


\section{INTRODUCTION}

Saccadic eye movements, which very quickly and accurately change the eye's position to a novel target, have been studied for many years in humans and animals. The various networks involved in their control have been localized and characterized by numerous experiments in awake animals using single cell recordings, as well as by behavioral investigations in animals, normal subjects, and patients using eye movement recordings, or functional studies using magnetic resonance imaging (fMRI) or transcranial magnetic stimulation (TMS) - for a review, see for instance ${ }^{1-4}$.

Moreover, the fact that eye movements can be recorded at a very high temporal and spatial resolution ${ }^{5,6}$, has been of particular benefit for investigators since reliable parameters of brain function can be deduced from such eye position tracks. Depending on the stimulus condition, these parameters may help to distinguish pathological conditions such as muscular/neuromuscular fatigue ${ }^{7-9}$ from various cortical and/or subcortical dysfunctions including lack of attention ${ }^{10-12}$, or the influence of motivation $^{13}$.

In particular, reflexive visually guided saccades can easily be elicited in a simple laboratory setting and their latency, velocity, as well as precision are highly reproducible with normal values varying little across the population. In addition, assessment of these eye movements is almost independent of individual cognitive (including language) skills, in contrast to neuropsychological test procedures.

These properties make the examination of (reflexive) visually guided saccades a promising method to objectively evaluate the effects of low-to-moderate alcohol intake levels of less than $0.8 \%$ [grams alcohol per $\mathrm{kg}$ body weight]. Such levels usually escape detection by conventional clinical examination, since typical symptoms and signs of inebriety including euphoria, decreased concentration, attention and/or perception, diminished ability to combine and adjust, increased spontaneity and talkativeness, and disturbances of equilibrium and visual perception, slowing of pupillary reaction, and pathological nystagmus ${ }^{14}$ are observed only at alcohol levels between $0.5 \%$ and $1.5 \%$.

Several studies have previously established that saccade latencies are prolonged and peak eye velocities are reduced under the influence of moderate-to-high levels of blood alcohol values ${ }^{15-18}$. Therefore, this study aimed at investigating the effects of low-dose alcohol intake, by analyzing whether reflexive visually evoked saccades, at alcohol levels below $0.8 \%$, become unambiguously pathological when compared to a normal population. If such an effect was shown, the evaluation of 
saccadic eye movements could be used in various applications related to work safety and/or for law enforcement officials to quickly assess a person's fitness to carry out sensitive tasks such as driving a vehicle. In traffic medicine, the development of performance criteria rather than individual threshold values for certain substances associated with the reduction of the ability to drive is of particular interest. Currently, according to Swiss legislation, any driver is deemed unfit to drive if the blood alcohol level exceeds $0.5 \%$. Although this level is fixed by the federal government, there is no defined threshold for fatigue or exhaustion which can likewise limit the ability to drive and/or perform similar sensitive tasks. Presently, the legal system does not rely on established threshold values linking illegal drugs or psycho-active drugs and their blood level concentration, respectively, to impairment of driving. Hence, any setting of a threshold for the intake of medicaments (dose, blood concentration, time between drug intake and driving) will be arbitrary. In contrast, a blood alcohol level of $0.5 \%$ is now accepted as a "reasonable" threshold in many countries. Thus, it seems appropriate to "calibrate" the influence of any pharmacologically active substance or sleep deprivation to alcohol. Political and legal acceptance of sanctions imposed on persons driving under the influence of a substance that has the same effect as $0.5 \%$ or more alcohol is expected to be given. Testing for abnormal saccadic eye movements would therefore represent a performance criterion with which abnormal patterns could be linked to a measured blood alcohol level.

\section{MATERIALS AND METHODS}

\section{Subjects}

Forty-one volunteers between 18 and 64 years of age were recruited by public announcement for participation in this study, and 40 subjects (24 males and 16 females) fully completed the test series. Only healthy, non-pregnant subjects were allowed to participate. The study was approved by the Ethical Committee of the University Hospital of Zürich (E03/2004) and adhered to the tenets of the Declaration of Helsinki. Informed, written consent was obtained from all participants.

\section{Time course}


All tests were performed in the late afternoon after a small lunch. An amount of alcohol expected to yield a blood alcohol level of at most $0.8 \%$, calculated according to Ulrich et al. ${ }^{19}$, was prepared. Subjects were asked to drink the alcohol in 5 to 10 minutes, and wash their mouth afterwards to ensure mouth alcohol did not influence the measurements. An alcohol breath test was administered immediately thereafter.

Eye movement recordings of visually guided saccades were performed before alcohol intake and at 15, 30, 45 and 60 minutes after intake. Measurements took approximately 3 minutes each. A venous blood sample was drawn from each subject at 15 and 45 minutes after alcohol intake and sent frozen in a heparin probe to a laboratory at the Institute of Legal Medicine at the University of Zurich. At 30 minutes, a small series of clinical tests were performed to evaluate divided attention: the Romberg test, in combination with estimating an interval of 30 seconds correctly, and a finger-to-nose test sequence that had to be repeated in correct order while standing on one leg and counting out loud from 20 to 50. Slow, smooth pursuit eye movements were evaluated as well as pupillary reaction and stance and gait. Test results were recorded as 0 (no effect), 1 (moderate effects like being talkative, bloodshot eyes) or 2 (clearly distinguishable deficits such as visibly disturbed gait, or disturbed equilibrium during the Romberg test or while standing on one leg). In addition, at 15, 30 and 45 minutes subjects were asked about the status of their subjective impairment. This self-evaluation was recorded as 1 (feeling normal), 2 (feeling some effect) or 3 (feeling a clear effect such as stating that he/she would no longer drive a motor vehicle).

\section{Eye movement recordings}

Saccadic eye movements were recorded using a video based infrared (IR) eye tracker device (OCULOMETRICS, Zurich, Switzerland), with a sampling rate of $500 \mathrm{~Hz}$ and a spatial resolution of $0.1^{\circ}$. This spatial resolution is comparable to that of the commonly used (but invasive) magnetic search coil technique ${ }^{5,6}$. Subjects were seated comfortably at a table with a soft chin rest. An LCD monitor (Belinea 101920, $60 \mathrm{~Hz}$ ) for presenting stimuli was positioned in front of them. A simple, visually guided pro-saccade paradigm was used to assess the state of reflexive eye movements. The target was presented on a black background at 5, 10, 15, and 20 degrees eccentricity to the right and left, with the same randomized sequence of trials for all sessions and subjects. Each trial began with a fixation period $(500 \mathrm{~ms})$ of a small center target $\left(0.8 \times 0.8^{\circ}\right)$. After successful fixation, the center target 
disappeared and a new stimulus was presented for $800 \mathrm{~ms}$. Subsequently, the trial ended with a dark screen of $200 \mathrm{~ms}$ duration. Each session consisted of 126 trials. After each recording session, the eye movement was evaluated by tracking both the pupil and the limbus and stored for offline analysis (see Schmitt et al. ${ }^{20}$ for details).

\section{Data analysis}

All data processing was performed offline using a commercial software package (MATLAB 7.7, The MathWorks Inc., Natick, MA). Saccades were detected automatically using combined velocity and acceleration criteria $^{21}$.

For each eccentricity, the latency (time lag between target presentation and onset of the saccadic eye movement), post-saccadic accuracy (difference between target position and eye position over an interval of $50 \mathrm{~ms}$ ), number of correct trials, and the so-called saccade main ${ }^{22-25}$ were determined. The main sequence characterizes the relationship between the peak velocity of the saccadic eye movement and the corresponding eye displacement. Thus, it is quantified by the peak eye velocity $\left(\mathrm{V}_{\max }\right)$ and a time constant $(\tau)$. The main sequence is determined over all eccentricities recorded in one session, that is, for each subject a main sequence is established after every eye test session recorded.

Since it was of interest to see whether saccadic parameters showed systematic abnormalities during alcohol exposure, the following statistical procedure was used: first, all individual latencies and mainsequence parameters were normalized. Second, data from an aged-matched group of normal subjects, available at the University Hospital of Zurich, was used as reference for normalization; this reference data was established in previous studies using the same paradigm and type of recordings. Finally, a one-way ANOVA with post-hoc test (Scheffé) was applied to both latencies and main sequences across all data; $p$-values of 0.05 were considered significant for all comparisons.

\section{RESULTS}

Achieved blood alcohol concentrations, subjective reporting, clinical testing 
Table 1 gives summary details of participants who completed the test procedures, the alcohol administered and the average blood alcohol concentrations (BAC) determined. In the first blood sample taken $15 \mathrm{~min}$. after alcohol intake, the average BAC was $0.43 \%$ ( \pm 0.17$)$; in the second sample an average of $0.54 \%$ ( \pm 0.12$)$ was determined. The BAC results are reported by their mean values only. Although the range for each subject was calculated by the laboratory, these values are omitted here for better readability.

TABLE 1: Summary of volunteer data (40 participants), administered alcohol and blood alcohol concentrations (BAC) determined.

\begin{tabular}{lcccccc}
\hline & \multicolumn{2}{c}{ Body } & Age & Alcohol & \multicolumn{2}{c}{ BAC } \\
& Weight [kg] & Height [cm] & [years] & ml of 96\% & BAC 1 & BAC 2 \\
\hline Average & 72.55 & 176.65 & 30.85 & 51.15 & 0.43 & 0.54 \\
\hline SD & 14.89 & 9.53 & 11.02 & 9.89 & 0.17 & 0.12 \\
\hline
\end{tabular}

As can be seen in Table 2, 3 subjects achieved a BAC of less than $0.2 \%$ in the first sample while 3 subjects had a BAC of more than $0.7 \%$. In the second sample, all subjects had a BAC above $0.2 \%$, in two subjects their BAC was over $0.7 \%$. None of the subjects had a BAC of more than $0.8 \%$.

TABLE 2: $\quad$ Achieved blood alcohol concentrations (BAC) as determined in the two blood samples taken per subject. 


\begin{tabular}{lc}
\hline & subjects \\
\hline less than $0.2 \%$ alcohol in first sample & 3 \\
\hline less than $0.2 \%$ alcohol in second sample & $\mathbf{0}$ \\
\hline more than $0.7 \%$ in first sample & 3 \\
\hline more than $0.7 \%$ in second sample & 3 \\
\hline & 5 \\
\hline number of subjects with decreasing BAC between & $\mathbf{5}$ \\
\hline nample 1 and 2 & $\mathbf{4 0}$ \\
\hline Total subjects & \\
\hline
\end{tabular}

The results of the self-evaluation are presented in Table 3. Most participants did feel a definite effect of alcohol already after $15 \mathrm{~min}$.

TABLE 3: Results of the self-evaluation given in counts and percentage (40 persons $=100 \%)$.

\begin{tabular}{l|cc|cc|cc}
\hline & \multicolumn{6}{|c}{ Response given by the subjects after } \\
\cline { 2 - 7 } & $\mathbf{1 5}$ minutes & {$[\%]$} & $\mathbf{3 0}$ minutes & {$[\%]$} & $\mathbf{4 5}$ minutes & {$[\%]$} \\
\hline Self-evaluation & 5 & 12.5 & 2 & 5 & 5 & 12.5 \\
feeling normal & 6 & 15 & 18 & 45 & 13 & 32.5 \\
feeling some effect & 29 & 72.5 & 20 & 50 & 21 & 52.5 \\
feeling a clear effect & & & & & 1 & 2.5 \\
n.a. & & & & & & \\
\hline
\end{tabular}

In the clinical examination performed 30min.after the end of alcohol consumption, 13 subjects did not show any signs of incapacitation despite alcohol fetor, while 18 subjects did show discrete signs 
(watery eyes, being more talkative). In 8 subjects alcohol intoxication was manifested clinically (stance/gait instability, difficulty standing on one leg and counting). One subject was not evaluated clinically.

\section{Saccadic eye movement parameters}

Figure 1 depicts a full set of data obtained from one subject (\#9) who volunteered to have 11 blood samples taken during 120 minutes after exposure to alcohol. Note the typical rise ( 40 min) and subsequent slow decline ( $0.1 \%$ per hour) of the BAC after quick intake of the body-mass adjusted $50 \mathrm{cc}$ of $96 \%$ alcohol. Note further that the latency at the start of the experiment $(203 \mathrm{~ms} \pm 6 \mathrm{~ms}$ [SD]) was clearly below the mean of the reference population (238ms $\pm 40 \mathrm{~ms}$ [SD]).

Figure 2 summarizes the normalized saccade parameters of all 40 subjects (group 1) and comparison with an aged-matched reference group of healthy subjects (group 2). After 75 minutes there were only five samples available which rendered those parameters obsolete; they are not included in the description of the results but merely shown for completeness.

\section{a) Accuracy}

Accuracy started to deteriorate immediately after alcohol exposure. A difference with regard to the reference group (group 2) was observed by the end of the BAC rise time, after which accuracy returned to normal levels with the decline of the BAC. However, there was no significant, lasting difference between the two groups.

\section{b) Latency}

On average, the saccade latency was slightly prolonged (mean $+1.2 \%$ ) during the BAC rise time yet consistently shorter (mean $-8.1 \%$ ) during BAC decline. Again, there was no significant difference either amongst group 1 across subjects nor when compared to group 2 at any time during the experiment.

c) Main sequence 
Both the peak eye velocity $\left(\mathrm{V}_{\max }\right)$ and the time constant $(\tau)$ declined immediately after alcohol intake by an average of $-0.2 \%$ (15 $\mathrm{min}$ ) and $-7.0 \%$ (after $15 \mathrm{~min}$ ) for $\mathrm{V}_{\max }$, respectively, and $-10 \%$ for $\tau$ (all times), clearly indicating the rapid decline of eye movement speed due to the alcohol. As with the other parameters, however, there was no significant difference during the experiment either between subjects in group 1 or between the two groups.

\section{DISCUSSION}

In close agreement with a number of earlier reports ${ }^{15-18}$, our study confirmed that visually evoked reflexive saccadic eye movements were markedly altered under the influence of alcohol. Latencies were slightly increased during the rise of the BAC level and shorter during its decline, whereas the main sequence parameters $\left(\mathrm{V}_{\max }, \tau\right)$ were clearly reduced at any time after exposure to alcohol.

However, the changes observed were not significantly, unambiguously, and consistently pathologic compared to an aged-matched population of normal and not inebriated controls, even if the subject felt impaired and deficiencies in a typical clinical test could be demonstrated. Whereas more than half of our subjects had a minor-to-obvious clinical finding, none of the saccade parameters obtained at the same time varied markedly different from the normal population. Visually evoked saccades are therefore not a parameter to unequivocally represent impairment due to low blood alcohol levels. It cannot be excluded that the influence on saccadic eye movements is significantly pronounced at higher doses of alcohol. However, this was not of interest in this study because signs and symptoms of alcohol influence manifest themselves quickly above $1.0 \%$ BAC.

Our experimental design required that the alcohol be ingested within a few minutes. By doing so, the subjective notion of the effect of alcohol was even stronger when compared to the same amount consumed over a longer time span. The discrepancy between the subjective feeling due to the effect of the rise of the BAC level and the objective measurements of the eye movement parameters was therefore expected.

As mentioned above, the fast intake of alcohol limits the power of the results of the self-evaluation. However, this limitation was accepted in order to design an experimental setting that allowed 
investigating the correlation of the BAC and parameters characterizing visually guided saccades in a standardized way.

In view of on-going discussions in the field of traffic medicine about defining performance criteria to assess the momentary ability to drive a vehicle, the results of this study must be interpreted in a legal context. Many countries have currently implemented maximum threshold values for substances such as alcohol which are tolerated in drivers. To ensure acceptance for the introduction of a new measure it seems mandatory that this new performance criterion is also reasonably well linked to currently established criteria. Hence, any new measure must be tested against threshold values currently accepted in society and law. The field of traffic and legal medicine needs to ensure that any new measures are not only scientifically sound, but also robust for legal application. While testing saccadic eye movements are a well-established measure in neurology, this study indicates that saccadic eye tests seem robust to low-dose alcohol intake.

In conclusion, our experiment showed that visually evoked saccadic eye movements are not altered by low levels of blood alcohol. Even when subjects felt impaired and significant clinical deficiencies were demonstrated consistently, pathologic parameters were not observed when comparing their saccadic performance with those of an aged-matched group of non-inebriated subjects. Therefore, reflexive visually evoked saccades are not suitable to clearly identify impairment due to low-dose alcohol intake.

\section{ACKNOWLEDGEMENTS}

The authors thank the Institute of Legal Medicine of the University of Zurich, Switzerland, for performing the blood alcohol analysis pro bono.

\section{COMPETING INTERESTS}

None. 


\section{FUNDING}

Funding was granted by the Swiss Road Safety Fund (Grant Number 305.03.01(2)).

\section{REFERENCES}

1 Carpenter, R. Movements of the eyes. London, UK: Pion Publ 1988.

2 Leigh R, Kennard C. Using saccades as a research tool in the clinical neurosciences. Brain 2006; 127:460-477.

3 Leigh R, Zee D. The neurology of eye movements. New York: Oxford University Press 1999.

4 Schwarz U. Neuroophthalmology: a brief vademecum. Eur J Radiol 2004;49:31-63.

5 Collewijn $\mathrm{H}$, van der Mark F, Jansen T. Precise recording of human eye movements. Vision Res 1975;15:447-450.

6 Robinson D. The mechanics of human saccadic eye movements. J Physio/ 1964;174:245-264.

7 Bahil. A, Stark L. Overlapping saccades and glissades are produced by fatigue in the saccadic eye movement system. Exp Neurol 1975;48:95-106.

8 Baloh R, Keesey J. Saccade fatigue and response to edrophonium for the diagnosis of myasthenia gravis. Ann N Y Acad Sci 1976;274:631-641.

9 Barton J, Jama A, Sharpe J. Saccadic duration and intrasaccadic fatigue in myasthenic and nonmyasthenic ocular palsies. Neurology 1995;45:2065-2072.

10 Gaymard B, Ploner C, Rivaud S et al. Cortical control of saccades. Exp Brain Res 1998;123:159163.

11 Pierrot-Deseilligny C, Rivaud S, Gaymard B et al. Cortical control of reflexive visually-guided saccades. Brain 1991;114(3):1473-1485.

12 Pierrot-Deseilligny C, Rivaud S, Gaymard B, et al. Cortical control of saccades. Ann Neurol 1995; 7:557-567.

13 Köhler G. Das Verhalten von Latenzzeiten saccadischer Augenbewegungen unter psychophysischen Beanspruchungen. München, Germany: Phd thesis, University of Munich 1979. 14 Schwerd W. Alkohol und Verkehrssicherheit. In Rechtsmedizin, Lehrbuch für Mediziner und Juristen, Köln, Germany: Deutscher Ärzteverlag 1992:110-23. 
15 Jantti V, Lang A, Keskinen E, et al. Acute effects of intravenously given alcohol on saccadic eye movements and subjective evaluations of intoxication. Psychopharmacology (Berl) 1983;79:251-255. 16 Lehtinen I, Lang A, Jantti V, et al. Acute effects of alcohol on saccadic eye movements. Psychopharmacology (Berl) 1979;63:17-23.

17 Nyberg S, Wahlstrom G, Backstrom T, et al. No difference in responsiveness to a low dose of alcohol between healthy women and men. Pharmacol Biochem Behav 2004;78:603-610.

18 Stapleton J, Guthrie S, Linnoila M. Effects of alcohol and other psychotropic drugs on eye movements: relevance to traffic safety. J Stud Alcohol 1986; 47:426-432.

19 Ulrich L, Cramer Y, Zink P. Calculating of blood alcohol values from alcohol intake using individual paramters. Blutalkohol 1987;24:192-198.

20 Schmitt KU, Muser M, Lanz C, et al. Comparing eye movements recorded by search coil and infrared eye tracking. J Clin Monit Comput 2007;21:49-53.

21 Schwarz U, Miles F. Ocular responses to translation and their dependence on viewing distance. I. Motion of the observer. J Neurophysiol 1991;66:851-864.

22 Bahill A, Clark M, Stark L. The main sequence, a tool for studying human eye movements. Math Biosci 1975;24:191-204.

23 Baloh R, Sills A, Kumley W, et al. Quantitative measurement of saccade amplitude, duration, and velocity. Neurology 1975;25:1065-1070.

24 Becker W. Metrics. In The neurobiology of saccadic eye movements. Reviews of oculomotor research, R.H. Wurtz and M.E. Goldberg, eds., New York: Elsevier, 1989:13-67.

25 Lebedev S, Van Gelder P, Tsui W. Square-root relations between main saccadic parameters. Invest Ophthalmol Vis Sci 1996;37:2750-2758. 


\section{FIGURE CAPTIONS}

FIG. 1: Data sampled from one subject (male, 48 years, $170 \mathrm{~cm}, 60 \mathrm{~kg}$ ), who agreed to have 11 blood samples taken during the time of the experiment (120min). After assessment of the baseline data, the subject was given $50 \mathrm{cc}$ of alcohol (at $0 \mathrm{~min})$. A: alcohol levels and time of eye movement recordings as well as clinical examinations. Black squares = blood alcohol level; open squares = breath alcohol level; small black squares = eye movement recording; open diamonds = clinical assessment; numbers denote the amount of correct saccades produced during the respective eye movement recording. $\mathbf{B}$ : mean saccade latencies $\pm 1 \mathrm{SE}$. C: main sequence parameters peak eye velocity $\left(\mathrm{V}_{\max }\right)$ and the time constant $(\tau)$. Black squares $=\mathrm{V}_{\max } \pm 95 \mathrm{Cl}$ (not showing up due to very small values!); white squares $=$ $\tau \pm 95 \mathrm{Cl}$ (note: values are multiplied by a factor of 100 to fit into the same axis).

FIG. 2: Synopsis of the normalized mean alcohol levels and saccade parameters of all subjects. Open squares $=$ mean $\pm 1 \mathrm{SD}$; gray patches $=$ mean $\pm 1 \mathrm{SD}$ of an aged-matched group of healthy people . 